\title{
VERLEIHUNG AUTORENPREIS DER SACAM
}

\section{Liebe Kolleginnen und Kollegen!}

Ein interessantes Thema in einen Artikel zu gießen ist eine schöne und erfüllende Arbeit. Man setzt sich mit der Materie manchmal schon Monate zuvor intensiv auseinander. Beim Schreiben kommt die Literatur-Recherche dazu. Diese führt immer zum eigenen Erkenntnis-Gewinn, ist aber auch mit einigem Aufwand verbunden. Die Zeitschrift „Akupunktur \& Aurikulomedizin“ hat mit dem ganzen Deutschsprachigen Raum eine große Reichweite. Eigene Erfahrungen und Erkenntnisse können so einem breiteren Publikum präsentiert werden.

Die Arbeit, die die Autoren leisten, ist eminent wichtig für die Wissensvermittlung unserer Mitglieder und ist für eine lebendige Gesellschaft unverzichtbar. Dies geschieht meistens neben einem vollen Praxis-Programm abends und an den Wochenenden. Um das zu würdigen, hat Mitgliederversammlung der SACAM 2020 beschlossen, einen Autorenpreis auszuloben. Erstmalig wird er für das diesjährige „Schweizer-Heft“, der zweiten Ausgabe des Jahres, vergeben. Die Jury besteht aus den Aurikulomedizinern im Vorstand. Sind Vorstandsmitglieder unter den Autoren, treten diese in den Ausstand.

Die Jury ist zu folgendem Ergebnis gekommen:

Die Arbeit von Franz Jost und Christine Spillmann mit dem Titel: „Achtung Herz!“ bekommt den dritten Preis in der Höhe von 200,- CHF.

Die Arbeit befasst sich mit der Möglichkeit, durch gezieltes Abfragen von Informationen am Ohr Herzkrankheiten antizipieren und behandeln zu können. Den Autoren gelingt es hierbei, eine Verbindung der klassischen Körperakupunktur und der Ohrakupunktur herzustellen und die Bedeutung der Meridianpunkte zum Schutz des Organs Herz hervorzuheben. Mit zwei Fallpräsentationen werden die Erkenntnisse (mit Erfolg) in die Praxis umgesetzt.

Der zweite Preis in der Höhe von 500,- CHF geht an Ruth Fiechter Boller für ihren Beitrag „Der Bahr-Meridian der Energie beim Hund, das seelische Trauma und die Bremer Stadtmusikanten - 2. Teil: Das seelische Trauma beim Tier".
Der erfahrenen Tierärztin gelingt es sehr gut, Verhaltensauffälligkeiten beiTieren (Hunde, Katzen, Kühe und Insekten) Punkten auf dem Energiemeridian nach Bahr zuzuordnen. Ihre Beobachtungen und Tests bei seelisch (emotional) traumatisierten Tieren und deren Bedeutung im klinischen Alltag werden analog zum Menschen pflanzlichen Urtinkturen zugeordnet und behandelt. Das Tier als Lebewesen erhält damit einen Wert an sich. Die Methode hilft uns, ein umfassenderes Verständnis für das Tier zu bekommen.

Der erste Preis in der Höhe von 800,- CHF geht an Christoph Scholtes für seinen Beitrag zu den „Aurikulomedizinischen Optionen bei Sars-CoV-2, speziell Long COVID“.

Dem Autor gelingt es hervorragend, schulmedizinische Erkenntnisse und Komplementärmedizin zu verbinden und Wege zu finden, Patienten mit stattgehabter COVID-Erkrankung zu helfen. Neue Erkenntnisse aus seiner Forschung im klinischen Alltag münden in neu beschriebenen Punkten am Ohr, die diagnostisch wie auch therapeutisch effektiven Nutzen bringen, speziell in Verbindung mit pflanzlichen Substanzen und Supplementen in Dosierungen, wie sie schulmedizinisch kritisiert und nicht empfohlen werden. Die Erfahrung zeigt aber, dass der von ihm aufgezeigte Weg wirkt und keine unkalkulierbaren Risiken birgt. Besonders hervorzuheben ist weiter, dass auch Menschen ohne Immunisierung für Sars-2-19 geholfen werden kann, was aber nicht als Freipass zur Impfverweigerung verstanden werden soll.

Den vierten und fünften Preis in der Höhe von je 200,- CHF teilen sich die folgenden Arbeiten:

- Ruth Fiechter-Boller: „Der Bahr-Meridian der Energie beim Hund, das seelische Trauma und die Bremer Stadtmusikanten - 3. Teil: Der Bahr-Energiemeridian und die Bremer Stadtmusikanten"

- Marianne Eggenberger: „Der interessante Fall“

Gute Gesundheit wünscht Ihnen Ihr

C. Scholtes

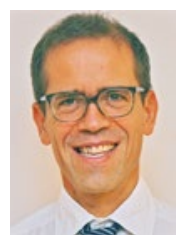

Dr. med. Christoph Scholtes

Co-Präsident der Schweizerischen Ärztegesellschaft für Akupunktur - Chinesische Medizin - Aurikulomedizin, SACAM

Wattenwylweg 21, 3006 Bern

Tel. $+41(0) 844200200$

info@sacam.ch,www.sacam.ch 